\title{
Proposta e validação de escala para a ferrugem alaranjada da cana-de-açúcar
}

\author{
Ana C. Klosowski, Lucimeris Ruaro, João C. Bespalhok Filho \& Louise L. May De Mio \\ Departamento de Fitotecnia e Fitossanitarismo, Setor de Ciências Agrárias, Universidade Federal do Paraná, 81531-990, \\ Curitiba, PR, Brasil
}

Autor para correspondência: Ana C. Klosowski, e-mail: ana_clkl@yahoo.com.br

\begin{abstract}
RESUMO
Objetivou-se neste trabalho elaborar e validar uma escala diagramática para a ferrugem alaranjada da cana-de-açúcar e comparar a sua eficiência em avaliar a doença com a escala da ferrugem marrom. Para elaboração da escala foram coletadas 120 folhas de uma cultivar suscetível à doença, e a maior e menor severidades encontradas representaram os extremos da escala. Os níveis intermediários foram calculados respeitando a lei de Weber-Fechner. A escala foi proposta com nove níveis de severidade: 0,$06 ; 0,14 ; 0,36 ; 0,89 ; 2,17$; 5,$18 ; 11,87 ; 24,92$ e 45,00\%. Oito avaliadores realizaram a validação estimando a severidade de 120 folhas em três avaliações: sem escala, com a escala da ferrugem marrom e com a escala da ferrugem alaranjada. A escala elaborada foi mais eficiente para avaliar a doença, proporcionando um coeficiente de determinação $\left(\mathrm{R}^{2}\right)$ médio de 0,91 e ausência de erros constantes e sistemáticos. Com a escala da ferrugem marrom, o $\mathrm{R}^{2}$ médio foi de 0,84 , e três avaliadores cometeram desvios constantes. Com a escala elaborada, aproximadamente $92 \%$ das estimativas estiveram na faixa de variação de $5 \%$ do valor real, enquanto que nas avaliações sem escala e com a escala da ferrugem marrom, aproximadamente $78 \%$ e $84 \%$ das estimativas, estiveram nessa faixa, respectivamente.
\end{abstract}

Palavras-chave: Puccinia kuehnii, Saccharum spp., patometria, severidade.

\section{ABSTRACT}

Proposal and validation of diagrammatic scale for assessment of orange rust of sugarcane

The aim of this work was to elaborate and validate a diagrammatic scale to quantify the severity of orange rust of sugarcane and to compare its efficiency in assessing the disease with the scale of brown rust. For the design of the scale, 120 sugarcane leaves were collected from a susceptible cultivar and the highest and the lowest severity founded were selected as extremes of scale. The intermediate levels were calculated according to the law of Weber-Fechner. The scale was proposed with nine levels of severity: 0,$06 ; 0,14 ; 0,36 ; 0,89 ; 2,17$; 5,$18 ; 11,87 ; 24,92$ and $45,00 \%$. The validation was accomplished by eight appraisers who estimated the severity of 120 leaves in three assessments: without scale, with scale of brown rust and with the proposed diagrammatic scale. The scale proposed was more efficient than the scale of brown rust to assess the severity of this disease, providing an average coefficient of determination $\left(R^{2}\right)$ of 0,91 and absence of constant and systematic errors. With the scale of brown rust, the average $\mathrm{R}^{2}$ was 0,84 and three appraisers committed constant errors. Moreover, with the scale proposed, approximately $92 \%$ of the estimates of the appraisers had been in the range of variation of $5 \%$ of the value of real severity, while in the assessments without scale and with scale of brown rust, approximately $78 \%$ and $84 \%$ of the estimates were in that range, respectively.

Key words: Puccinia kuehnii, Saccharum spp., pathometry, severity.

A ferrugem alaranjada, causada pelo fungo Puccinia kuehnii (W. Krüger) E.J. Butler, foi detectada em dezembro de 2009 no Estado de São Paulo (Barbasso et al., 2010) e já está presente em outras áreas produtoras da cana-de-açúcar como o Paraná, Espírito Santo, Minas Gerais, Goiás e Mato Grosso do Sul (CTC, 2011). Devido ao grande prejuízo que causou nos canaviais australianos no ano de 2000, chegando a diminuir a produção em $24 \%$ em toneladas de Pol por hectare (TPH) (Braithwaite, 2005), e ao rápido avanço no território nacional, a doença chegou causando alarde dentre os produtores da cultura no Brasil.

A quantificação de doenças é importante para que se desenvolvam medidas de controle e para que se estudem as suas variáveis epidemiológicas (Bergamin Filho \& Amorim, 1996). Como os sintomas da ferrugem alaranjada aparecem em forma de lesões no limbo foliar, a melhor maneira de estimar a sua severidade é calculando-se a porcentagem de área foliar que apresenta os sintomas da doença em relação à área foliar total.

Essa avaliação de severidade é normalmente subjetiva, pois é estimada visualmente e pode variar entre os avaliadores. A escala diagramática pode diminuir essa subjetividade, aumentando a acurácia e a precisão das estimativas dentre os diferentes avaliadores, como já demonstrado em outros patossistemas (Martins et al., 2004; Kowata et al., 2008; Salgado et al., 2009; Sachs et al., 2011). No entanto, esta escala deve ser de fácil uso, aplicada a diferentes condições e idades da planta e produzir resultados reproduzíveis.

Os sintomas da ferrugem alaranjada se caracterizam pela formação de pústulas pequenas e ovais de cor alaranjada 
a castanho-alaranjada no limbo foliar que, mesmo em condições de menores severidades, tendem a apresentar um padrão coalescido, ou seja, as lesões se agrupam. Essa é a sua principal diferença em relação à ferrugem marrom (Puccinia melanocephala Syd. et P. Syd.), na qual as lesões, que são maiores e mais arredondadas, só coalescem em altas severidades (Magarey, 2000; Infante et al., 2009). A diferença entre os sintomas das duas doenças pode ser observada na Figura 1.
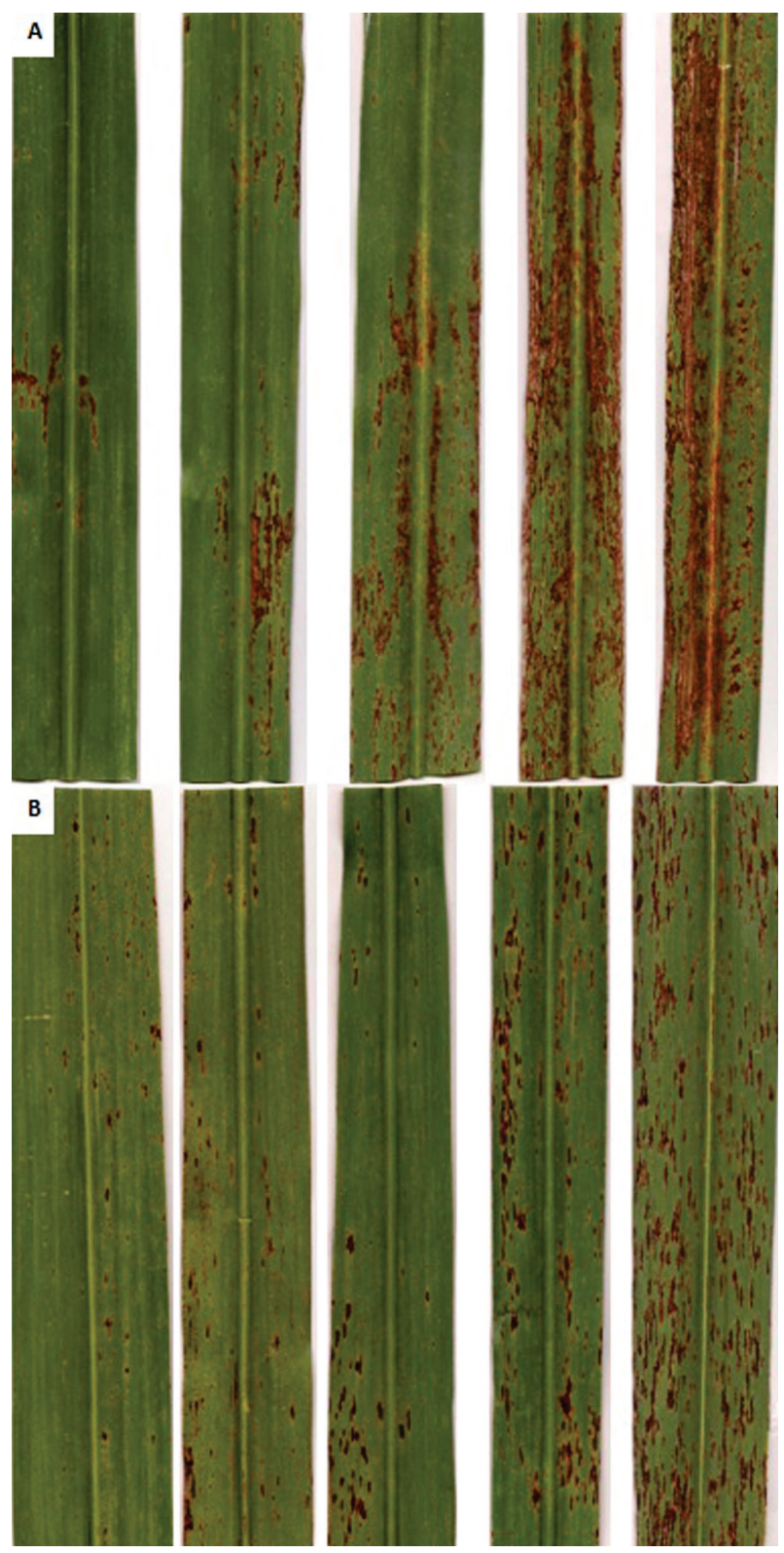

FIGURA 1 - A. Sintomas da ferrugem alaranjada, causada pelo fungo Puccinia kuehnii, em folhas de cana-de-açúcar (Saccharum spp.); B. Sintomas da ferrugem marrom, causada pelo fungo Puccinia melanocephala, em folhas de cana-de-açúcar (Saccharum spp.).
As diferenças no padrão dos sintomas das duas doenças se constituem na justificativa da construção de uma escala diagramática específica para avaliar a severidade da ferrugem alaranjada, pois o uso da escala da ferrugem marrom (Amorim et al., 1987), que tem sido utilizada para avaliar a ferrugem alaranjada desde a sua chegada no Brasil, pode comprometer a exatidão das estimativas desta doença, por não representar fielmente o sintoma observado no campo.

Com base no contexto descrito, este trabalho teve como objetivos elaborar uma escala diagramática para a ferrugem alaranjada da cana-de-açúcar e realizar a validação desta e da escala diagramática da ferrugem marrom (Amorim et al., 1987), a fim de comparar a eficiência das duas escalas para avaliação de severidade da ferrugem alaranjada.

Foram coletadas 120 folhas de cana-de-açúcar da cultivar RB 72454, (suscetível à ferrugem alaranjada), com diferentes níveis de severidade da doença em campo instalado na Estação Experimental da Universidade Federal do Paraná, em Paranavaí-PR. Para a determinação da severidade real, um segmento de $25 \mathrm{~cm}$ da folha foi escaneado com auxílio de uma impressora multifuncional $4280 \mathrm{HP}$. Estas folhas foram impressas e as lesões foram copiadas com uma caneta de tinta permanente em um papel de transparência para posterior medição da área total de lesões em cada folha. Essa medição foi realizada no equipamento Winrhizo v.4.0, Regent Systems, Quebec, (Canadá), acoplado a um scanner, utilizando a definição de 400 dpi. A área total de cada folha foi medida com auxílio do mesmo equipamento. Com esses dados foi calculada a severidade real de cada folha. Para a elaboração da escala, determinou-se uma área foliar padrão e selecionou-se a maior e a menor severidade, em porcentagem, encontradas no campo, como extremos da escala. Então, determinaramse os níveis intermediários, de acordo com a lei de WeberFechner da acuidade visual (Horsfall \& Barratt, 1945).

Foram selecionados oito avaliadores, dentre os quais, quatro com experiência na avaliação de doenças utilizando escalas diagramáticas e quatro sem experiência, que receberam treinamento prévio em programa de computador para treinamento de avaliadores de severidade de doenças. Primeiramente, todos os avaliadores estimaram a severidade da doença, em porcentagem, para cada uma das 120 folhas organizadas de forma aleatória quanto à severidade, sem o uso da escala. Então, avaliaram as mesmas folhas com auxílio da escala diagramática da ferrugem marrom da cana-de-açúcar (Amorim et al., 1987) e, por último, fizeram a mesma avaliação com auxílio da escala diagramática elaborada para a ferrugem alaranjada.

A acurácia e a precisão das estimativas visuais dos avaliadores foram determinadas por regressão linear simples, considerando a severidade real como variável independente e a severidade estimada como variável dependente. A acurácia das estimativas foi determinada pelo teste $t$ aplicado ao intercepto da regressão linear (a), para verificar se foi significativamente diferente de 0 , e ao coeficiente angular 
da reta (b), para testar se foi significativamente diferente de 1 , ao nível de $5 \%$ de probabilidade. Valores de intercepto diferentes de 0 indicam a presença de desvios constantes, enquanto valores de coeficiente angular da reta que desviam de 1 indicam a presença de desvios sistemáticos.

A precisão das estimativas foi avaliada pelo coeficiente de determinação da regressão $\left(\mathrm{R}^{2}\right)$ e pela variância dos erros absolutos (severidade estimada menos severidade real). Para fins de avaliação dos resíduos, foram adotados os critérios utilizados nos programas de computador para treinamento de avaliadores de severidade de doenças como Distrain (Tomerlin \& Howell, 1988), Disease.Pro (Nutter Jr. \& Worawitlikit, 1989) e Helmap (Canteri et al., 1999), os quais classificam como excelente, um avaliador cujos erros sejam menores que $5 \%$, e bom, aquele cujos erros não ultrapassem $10 \%$. Além disso, comparou-se o desvio máximo que cada avaliador cometeu indicando, em valor absoluto, a diferença da sua estimativa mais distante do valor real da severidade.

A escala diagramática elaborada neste trabalho foi mais eficiente para avaliar a severidade da ferrugem alaranjada que a escala diagramática da ferrugem marrom (Amorim et al., 1987), atingindo um $\mathrm{R}^{2}$ médio de 0,91, e podendo ser indicada para utilização em programas de melhoramento e em estudos epidemiológicos da doença.

A escala proposta apresenta nove níveis de severidade e tem como limite inferior e superior os valores de $0,06 \%$ e $45 \%$ de área lesionada, respectivamente (Figura 2).

Os valores do intercepto foram diferentes de $0(\mathrm{p} \leq 5)$ para $50 \%$ dos avaliadores na avaliação sem o uso de escala e, para 38\%, na avaliação com auxílio da escala da ferrugem marrom (Tabela 1). Já com o uso da escala da ferrugem alaranjada, todos os avaliadores obtiveram valores de intercepto estatisticamente iguais a $0(\mathrm{p} \leq 5)$, o que significa que nenhum avaliador cometeu desvios constantes. $\mathrm{Na}$ avaliação sem escala, para cada $1 \%$ de incremento da severidade real da doença, houve $2,96 \%$ de incremento na estimativa feita pelos avaliadores sem experiência e 1,56\% na estimativa feita pelos avaliadores com experiência. $\mathrm{Na}$ avaliação com a escala da ferrugem marrom, os avaliadores sem experiência estimaram, em média, um incremento de $2,44 \%$ a cada $1 \%$ de incremento da severidade real, e dentre aqueles com experiência, apenas o avaliador 8 apresentou uma tendência em superestimar a doença.

Os valores do coeficiente angular da reta não diferiram de $1(\mathrm{p} \leq 5)$ para todos os avaliadores, tanto na avaliação sem
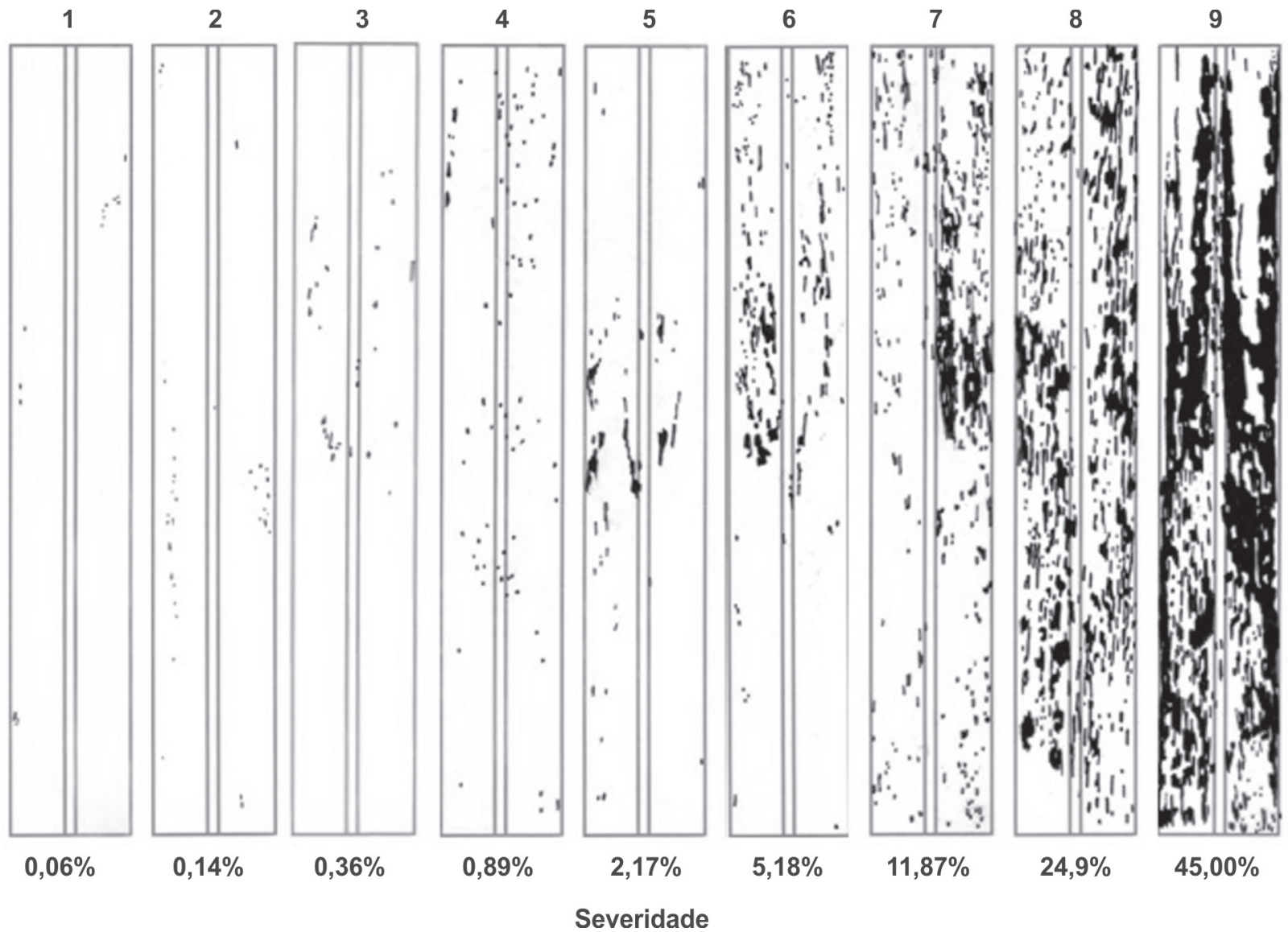

FIGURA 2 - Escala diagramática para avaliação de severidade da ferrugem alaranjada, causada pelo fungo Puccinia kuehnii em folhas de cana-de-açúcar (Saccharum spp.). Valores em porcentagem de área foliar lesionada. 
escala, quanto nas avaliações com ambas as escalas (Tabela 1). Isso indica a ausência de desvios sistemáticos.

Quanto à precisão dos avaliadores, as estimativas visuais de severidade, na avaliação sem o uso de escala, explicaram 69 a 90\% $\left(\mathrm{R}^{2}\right)$ da variação na medida da severidade real da doença, e a média dos avaliadores foi de $82 \%$ (Tabela 1). Na avaliação com auxílio da escala da ferrugem marrom, as estimativas visuais explicaram 77 a $90 \%\left(\mathrm{R}^{2}\right)$ da variação na medida da severidade real, sendo a média dos avaliadores de $84 \%$. Na avaliação com auxílio da escala da ferrugem alaranjada as estimativas visuais de severidade explicaram 87 a 94\% ( $\left.\mathrm{R}^{2}\right)$ da variação na medida da severidade real, sendo a média geral dos avaliadores igual a 91\% (Tabela 1). A média dos avaliadores com experiência foi de $89 \%$ e, dos sem experiência, foi de 93\%. Neste caso, $75 \%$ dos avaliadores apresentaram um coeficiente de determinação $\left(\mathrm{R}^{2}\right)$ maior que $90 \%$, o que é bastante desejado na validação de uma escala diagramática. Portanto, os avaliadores conseguiram uma precisão alta com o uso da escala elaborada neste trabalho, o que pode ser explicado pela identificação dos sintomas encontrados nas folhas com o padrão daqueles apresentados nesta escala.

As diferenças entre os valores estimados visualmente e os valores reais de severidade (resíduos), nas avaliações sem e com ambas as escalas, concentraram-se na faixa de $10 \%$ para mais ou para menos (Tabela 2 ), sendo que menos de 5,3 e $1 \%$ das estimativas estiveram fora dessa faixa na avaliação sem escala, com a escala da ferrugem marrom e com a escala da ferrugem alaranjada, respectivamente.

$\mathrm{Na}$ avaliação sem a escala, 96,58\% das estimativas dos avaliadores sem experiência ficou dentro da faixa de variação de $10 \%$, enquanto que para os avaliadores com

TABELA 1 - Coeficientes lineares (a), angulares (b) e de correlação $\left(\mathrm{R}^{2}\right)$, obtidos nas regressões entre severidade real e estimada da doença, a partir de folhas de cana-de-açúcar (Saccharum spp.) com sintomas de ferrugem alaranjada causada pelo fungo Puccinia kuehnii, em avaliações sem auxílio de escala, com auxílio da escala de Amorim et al. (1987) e com o uso da escala elaborada para a ferrugem alaranjada

\begin{tabular}{|c|c|c|c|c|c|c|c|c|c|c|c|c|}
\hline \multirow[t]{2}{*}{ Avaliação } & \multirow[t]{2}{*}{ Coeficientes } & \multicolumn{8}{|c|}{ Avaliadores } & \multicolumn{3}{|c|}{ Média } \\
\hline & & 1 & 2 & 3 & 4 & 5 & 6 & 7 & 8 & $\begin{array}{c}\text { Sem } \\
\text { experiência }\end{array}$ & $\begin{array}{c}\text { Com } \\
\text { experiência }\end{array}$ & Geral \\
\hline \multirow[t]{3}{*}{ Sem escala } & $\mathrm{a}$ & $4,86^{*}$ & $2,41 *$ & 1,21 & $3,37 *$ & $-0,11$ & $-0,88$ & $-0,39$ & $6,84 *$ & $2,96^{*}$ & $1,56^{*}$ & $4,60^{*}$ \\
\hline & $\mathrm{b}$ & 1,34 & 0,94 & 0,83 & 1,13 & 1,10 & 1,15 & 0,47 & 1,66 & 1,06 & 1,10 & 1,08 \\
\hline & $\mathrm{R}^{2}$ & 0,87 & 0,85 & 0,69 & 0,90 & 0,88 & 0,79 & 0,79 & 0,83 & 0,83 & 0,82 & 0,82 \\
\hline \multirow{3}{*}{$\begin{array}{l}\text { Escala de } \\
\text { Amorim et al. } \\
\text { (1987) }\end{array}$} & $\mathrm{a}$ & $4,26^{*}$ & $2,77^{*}$ & 1,66 & 1,07 & $-0,19$ & 0,15 & $-0,87$ & $4,62 *$ & 1,68 & $2,44 *$ & 0,93 \\
\hline & $\mathrm{b}$ & 1,10 & 1,24 & 1,00 & 1,12 & 1,10 & 0,89 & 0,76 & 1,59 & 1,10 & 1,12 & 1,09 \\
\hline & $\mathrm{R}^{2}$ & 0,85 & 0,83 & 0,85 & 0,90 & 0,84 & 0,82 & 0,77 & 0,85 & 0,86 & 0,82 & 0,84 \\
\hline \multirow{3}{*}{$\begin{array}{l}\text { Escala da } \\
\text { ferrugem } \\
\text { alaranjada }\end{array}$} & $\mathrm{a}$ & 1,53 & 1,10 & 1,71 & 0,98 & $-0,64$ & 0,17 & 0,66 & 0,75 & 1,58 & 0,24 & 0,91 \\
\hline & $\mathrm{b}$ & 1,04 & 0,95 & 0,83 & 0,76 & 0,99 & 0,80 & 0,92 & 0,76 & 0,90 & 0,87 & 0,88 \\
\hline & $\mathrm{R}^{2}$ & 0,94 & 0,93 & 0,92 & 0,92 & 0,93 & 0,88 & 0,87 & 0,90 & 0,93 & 0,89 & 0,91 \\
\hline
\end{tabular}

*significativo a $5 \%$ de probabilidade.

TABELA 2 - Desvio máximo em valor absoluto (D.M.V.A.), valores na faixa de variação de 10\% (valores $=\mathrm{x} \pm 10$ ) e valores na faixa de variação de $5 \%$ (valores $=\mathrm{x} \pm 5$ ) das estimativas dos avaliadores em relação ao valor de severidade real de folhas de cana-de-açúcar (Saccharum spp.) com sintomas de ferrugem alaranjada causada pelo fungo Puccinia kuehnii, sem auxílio de escala, com auxílio da escala de Amorim et al. (1987) e com o uso da escala elaborada para a ferrugem alaranjada

\begin{tabular}{|c|c|c|c|c|c|c|c|c|c|c|c|c|}
\hline \multirow[t]{2}{*}{ Avaliação } & \multirow[t]{2}{*}{ Parâmetros } & \multicolumn{8}{|c|}{ Avaliadores } & \multicolumn{3}{|c|}{ Média } \\
\hline & & 1 & 2 & 3 & 4 & 5 & 6 & 7 & 8 & $\begin{array}{c}\text { Sem } \\
\text { experiência }\end{array}$ & $\begin{array}{c}\text { Com } \\
\text { experiência }\end{array}$ & Geral \\
\hline \multirow{3}{*}{$\begin{array}{l}\text { Sem } \\
\text { escala }\end{array}$} & D.M.V.A. & 12,38 & 14,90 & 23,44 & 12,95 & 11,55 & 16,75 & 9,26 & 21,50 & 15,92 & 14,77 & 15,34 \\
\hline & $\%$ Valores $=\mathrm{x} \pm 10$ & 95,89 & 97,26 & 94,52 & 98,63 & 95,89 & 91,78 & 100,00 & 86,30 & 96,58 & 93,49 & 95,03 \\
\hline & $\%$ Valores $=\mathrm{x} \pm 5$ & 75,34 & 86,30 & 78,08 & 87,67 & 83,56 & 76,71 & 86,30 & 50,68 & 81,85 & 74,31 & 78,08 \\
\hline \multirow{3}{*}{$\begin{array}{l}\text { Escala de } \\
\text { Amorim et } \\
\text { al. (1987) }\end{array}$} & D.M.V.A. & 13,65 & 12,12 & 11,37 & 15,34 & 10,82 & 14,91 & 9,15 & 18,35 & 13,12 & 13,31 & 13,21 \\
\hline & $\%$ Valores $=\mathrm{x} \pm 10$ & 95,89 & 97,26 & 98,63 & 98,63 & 98,63 & 97,26 & 100,00 & 93,15 & 97,60 & 96,26 & 97,43 \\
\hline & $\%$ Valores $=\mathrm{x} \pm 5$ & 83,56 & 84,93 & 90,41 & 86,30 & 91,78 & 87,67 & 90,41 & 56,16 & 87,87 & 81,51 & 83,90 \\
\hline \multirow{3}{*}{$\begin{array}{l}\text { Escala da } \\
\text { ferrugem } \\
\text { alaranjada }\end{array}$} & D.M.V.A. & 7,00 & 7,75 & 7,76 & 5,90 & 7,40 & 10,20 & 11,10 & 9,52 & 7,10 & 9,56 & 8,33 \\
\hline & $\%$ Valores $=\mathrm{x} \pm 10$ & 100,00 & 100,00 & 100,00 & 100,00 & 100,00 & 98,63 & 98,63 & 100,00 & 100,00 & 99,32 & 99,66 \\
\hline & $\%$ Valores $=\mathrm{x} \pm 5$ & 94,52 & 94,52 & 89,04 & 94,52 & 87,67 & 93,15 & 95,89 & 94,52 & 91,39 & 90,41 & 91,78 \\
\hline
\end{tabular}


experiência esse valor foi de 93,49\% (Tabela 2). Com auxílio da escala da ferrugem marrom, $97,60 \%$ e $96,26 \%$ das estimativas dos avaliadores sem e com experiência, respectivamente, estiveram na faixa da variação de $10 \%$. Com o uso da escala da ferrugem alaranjada, os resultados foram melhores, sendo que todas as estimativas dos avaliadores sem experiência estiveram na faixa de variação de $10 \%$ e apenas duas estimativas, uma do avaliador 6 e outra do avaliador 7, ficaram fora dessa faixa de erro para os avaliadores com experiência. Com o uso da escala da ferrugem alaranjada, a porcentagem de valores dentro da faixa de variação de $5 \%$ também foi maior, o que indica que as estimativas dos avaliadores foram mais próximas ao valor real da severidade quando eles tiveram auxílio desta escala.

O desvio máximo da severidade real em valor absoluto baixou com o uso da escala da ferrugem alaranjada, correspondendo a um desvio máximo 55\% menor para os avaliadores sem experiência e 35\% menor para os avaliadores com experiência, em relação à avaliação sem escala. Quando se compara com a avaliação com a escala da ferrugem marrom, a escala da ferrugem alaranjada proporcionou um desvio máximo $46 \%$ menor para os avaliadores sem experiência e $28 \%$ menor para os avaliadores com experiência.

Os resultados da validação mostraram que a escala da ferrugem marrom proporcionou aos avaliadores acurácia e precisão semelhantes àquelas obtidas sem o uso de qualquer escala. Em alguns casos, com o uso desta escala, alguns avaliadores apresentaram um desempenho pior em relação à avaliação sem escala, como ocorreu em relação ao coeficiente de determinação $\left(\mathrm{R}^{2}\right)$ dos avaliadores 1,2 , 5 e 7. Ao contrário, observou-se que a escala da ferrugem alaranjada proporcionou melhores acurácia e precisão em relação às duas avaliações mencionadas e, com o seu auxílio, os avaliadores obtiveram valores satisfatórios em todos os parâmetros avaliados.

Os dados obtidos na regressão dos avaliadores sem experiência demonstraram que o treinamento foi efetivo para aumentar sua acurácia e precisão, sendo que, em vários parâmetros, estes mostraram maior habilidade em avaliar a doença que os avaliadores com experiência. Isso é justificável pelo fato de o treinamento ter sido realizado com folhas e sintomas semelhantes à ferrugem alaranjada na cana-de-açúcar, e a experiência dos avaliadores, muitas vezes, estar relacionada a doenças e culturas muito diferentes desta. No entanto, ambas as classes apresentaram resultados satisfatórios na validação das escalas.

As observações de Nutter \& Schultz (1995) quanto à variação na habilidade entre indivíduos para discriminar níveis de doença pode explicar a diferença entre os avaliadores nas estimativas de severidade da ferrugem alaranjada da cana-de-açúcar. Mesmo com o uso de uma escala, fatores inerentes ao indivíduo como cansaço, fadiga, humor, entre outros, podem influenciar a acurácia e precisão da estimativa.
A escala diagramática proposta para ferrugem alaranjada mostrou-se mais adequada que a escala diagramática da ferrugem marrom (Amorim et al., 1987) para a avaliação de severidade da ferrugem alaranjada da cana-de-açúcar, permitindo quantificar os sintomas de forma mais acurada e precisa.

\section{AGRADECIMENTOS}

Os autores agradecem aos alunos de Pós-Graduação em Agronomia - Produção Vegetal da Universidade Federal do Paraná - UFPR, que atuaram como avaliadores na validação da escala.

\section{REFERÊNCIAS BIBLIOGRÁFICAS}

Amorim L, Bergamin Filho A, Cardoso C, Moraes VA, Fernandes CR (1987) Metodologia de avaliação da ferrugem da cana-deaçúcar (Puccinia melanocephala). Boletim Técnico Copersucar 39:13-16.

Barbasso D, Jordão H, Maccheroni W, Boldini J, Bressiani J, Sanguino A (2010) First report of Puccinia kuehnii, causal agent of orange rust of sugarcane, in Brazil. Plant Disease 94:1170.

Bergamin Filho A, Amorim L (1996) Doenças de plantas tropicais: epidemiologia e controle econômico. São Paulo SP. Agronômica Ceres.

Braithwaite K (2005) Assessing the impact that pathogen variation has on the sugarcane breeding program. Final report SRDC project BSS258. BSES Limited Publication. Brisbane, Australia.

Canteri MG, Dalla-Pria M, Nascimento MA (1999) Helmap software para seleção e treinamento de avaliadores de severidade de doenças das culturas do milho e da cana-de-açúcar. Ponta Grossa PR. Universidade Estadual de Ponta Grossa.

CTC - Centro de Tecnologia Canavieira (2011) Mapa das ocorrências da ferrugem alaranjada. Disponível em: www. ctcanavieira.com.br/index.php?option $=$ com_content\&view $=$ ar ticle\&id=507:ferrugem-alaranjada-da-cana-de-acucar-mapa-de ocorrencias\&catid=3:destaque $\&$ Itemid $=1460$. Acesso em 20 de outubro de 2011.

Horsfall JG, Barratt RW (1945) An improved grading system for measuring plant disease. Phytopathology 35:655.

Infante D, Martinez B, Gonzalez E, Gonzalez N (2009) Puccinia kuehnii (Kruger) Butler y Puccinia melanocephala H. Sydow y P. Sydow en el cultivo de la caña de azúcar. Revista Protección Vegetal 24:22-28.

Kowata LS, May De Mio LL, Dalla-Pria M, Santos HAA (2008) Escala diagramática para avaliar severidade de míldio na soja. Scientia Agraria 9:105-110.

Magarey RC (2000) Orange rust. In: Rott P, Bailey RA, Comstock JC, Croft BJ, Girard JC, Saumtally AS (Eds.) A guide to sugarcane diseases. Montpellier França. La Librairie du Cirad. pp. 121-125.

Martins MC, Guerzoni RA, Câmara GMS, Mattiazzi P, Lourença SA, Amorim L (2004) Escala diagramática para a quantificação do complexo de doenças foliares de final de ciclo em soja. Fitopatologia Brasileira 29:179-184.

Nutter Jr. FW, Worawitlikit O (1989) Disease.Pro: a computer 
program for evaluating and improving a person ability to assess disease proportion. Phytopathology 79:1135.

Nutter Jr. FW, Schultz PM (1995) Improving the accuracy and precision of disease assessments: selection of methods and use of computer-aided training programs. Canadian Journal of Plant Pathology 17:174-184.

Sachs PJD, Neves CSVJ, Canteri MG, Sachs LG (2011) Escala diagramática para avaliação da severidade da mancha branca em milho. Summa Phytopathologica 37:202-204.

Salgado M, Pozza EA, Lima LM, Pereira RTG, Pfenning LH (2009) Escala diagramática para avaliação da severidade da mancha de Phoma do cafeeiro. Tropical Plant Pathology 34:422-427.

Tomerlin JR, Howell TA (1988) Distrain: a computer program for training people to estimate disease severity on cereal leaves. Plant Disease 72:455-459.

TPP 544 - Recebido 14 Março 2012 - Aceito 1 Novembro 2012

Editor de Seção: Lilian Amorim 